\title{
Shot-noise suppression by Fermi and Coulomb correlations in ballistic conductors
}

\author{
O. M. Bulashenko and J. M. Rubí \\ Departament de Física Fonamental, Universitat de Barcelona, Diagonal 647, E-08028 Barcelona, Spain
}

(Received 30 June 2000; revised manuscript received 12 January 2001; published 20 June 2001)

\begin{abstract}
We investigate the injection of degenerate Fermi-Dirac electrons into a multimode ballistic conductor under the space-charge-limited regime. The nonequilibrium current fluctuations were found to be suppressed by both Coulomb and Fermi correlations. We show that the Fermi shot-noise-suppression factor is limited below by the value $2 k_{B} T / \varepsilon_{F}$, where $T$ is the temperature and $\varepsilon_{F}$ the Fermi energy of the injected electrons. The Coulomb noise suppression factor may attain much lower values $\varepsilon_{F} / 2 q U$, because of its dependence on the applied bias $U \gg k_{B} T / q$. The asymptotic behavior of the overall shot-noise suppression factor in a high degenerate limit was found to be $k_{B} T / q U$, independent of the material parameters.
\end{abstract}

DOI: 10.1103/PhysRevB.64.045307

PACS number(s): 73.50.Td

\section{INTRODUCTION}

Nonequilibrium fluctuations of the electric current (shot noise) in mesoscopic conductors have received recently significant attention. ${ }^{1,2}$ In particular, the shot noise in scatteringfree or ballistic conductors has been studied extensively both theoretically ${ }^{3-5}$ and experimentally, ${ }^{6-9}$ by focusing mainly on the suppression of noise by Fermi correlations in quantum point contacts under low temperatures, i.e., conductors with a small number of quantum modes.

On the other hand, when the ballistic transport is limited by a space charge, Coulomb correlations may also result in a shot-noise suppression. If the electron density injected into a ballistic conductor is low, the electron gas is nondegenerate, and Fermi statistical correlations are not efficient. For this case the Coulomb correlations are the main source of the shot-noise suppression, as has been demonstrated by Monte Carlo simulations, ${ }^{10,11}$ and subsequently analytically in a framework of the Vlasov system of equations. ${ }^{12}$ In nanoscale devices, however, the injected carriers are usually degenerate, which is due to a high level of contact doping and the elevated position of the Fermi level in the contact emitter. Therefore, it is of interest to consider the situation when both mechanisms, Fermi and Coulomb correlations, act together-the case that is important not only from a fundamental, but also from an applied point of view ${ }^{13}$ and has attracted less attention so far. ${ }^{14,15}$ In Ref. 14 the problem for a multimode degenerate conductor in the presence of a nearby gate has been posed, and the numerical results has been presented for a two-dimensional field-effect-transistor geometry. Monte Carlo simulations in a two-terminal geometry, which take into account the degenerate injection from the contacts and Coulomb correlations in the ballistic region, have been performed. ${ }^{15}$ The relative significance of each mechanism in the shot-noise suppression and the limiting values for the noise suppression factors of each mechanism still remain unclear, since the analytical theory has not been proposed.

It is the objective of the present paper to address the problem of shot-noise suppression under the conditions of the interplay between Fermi and Coulomb correlations in twoterminal multimode ballistic conductors. To this purpose, we apply the recently developed analytical theory ${ }^{16}$ for a spacecharge-limited (SCL) ballistic conductors to the case of a Fermi-Dirac degenerate injection. Since we address the case of thick (in transversal dimensions) samples, the number of transversal modes (quantum channels) is large and the dimensionality of a momentum space of electrons is threedimensional (3D), which makes a difference with the previous considerations of a one-channel or a few-channel quantum ballistic conductor (1D or quasi-1D momentum space). ${ }^{2-5}$ Our analysis goes beyond the linear-response regime and zero-temperature limit-the assumptions typically used to study few-channel conductors. ${ }^{2-5}$ In a semiclassical framework, for a multimode ballistic conductor, we have derived analytical formulas that determine the nonlinear $I-V$ characteristics, the current-noise spectral density, and the shot-noise suppression factors for each suppression mechanism in the limit of high biases. We show that the Fermi shot-noise-suppression factor is limited below by the value determined by the properties of the injecting contact (the ratio between the temperature and the Fermi energy), whereas the Coulomb noise suppression may be enhanced arbitrarily strong by extending the length of the ballistic sample with a simultaneous increase of bias (provided the transport remains ballistic). Therefore, the Coulomb suppression may be achieved much stronger even in samples with a high degree of an electron degeneracy.

The paper is organized as follows. In Sec. II we describe the semiconductor structure under consideration and discuss the main assumptions concerning the model. In Sec. III we introduce the electron distribution function over the longitudinal injection energy, found by integrating over the transversal modes. The analytical expression for the mean current is derived as a function of the self-consistent potential barrier height. Then, in the limit of high biases, the current-voltage characteristics beyond the Child approximation is obtained, which takes into account the degenerate Fermi-Dirac injection. In Sec. IV the analytical expression for the suppressed value of the shot-noise power is derived, in which the Fermiand Coulomb-correlation contributions are distinguished. The results for a particular GaAs semiconductor SCL diode are presented in Sec. V. Finally, Sec. VI summarizes the main conclusions of the paper. 


\section{THE PHYSICAL MODEL}

We consider a two-terminal semiconductor ballistic sample with plane-parallel heavily doped contacts at $x=0$ and $x=l$. The structure may be considered as a $n-i-n$ SCL homodiode $^{12}$ in which the current is determined by a charge injection from the contacts rather than by intrinsic carriers of the ballistic region. The applied bias $U$ between the contacts is assumed to be fixed by a low-impedance external circuit and does not fluctuate. In order to simplify the problem, we assume that due to the large difference in the carrier density between the contacts and the sample, and hence in the corresponding Debye screening lengths, all the band bending occurs in the ballistic base, and the relative position of the conduction band and the Fermi level does not change in the contacts. Therefore, when the bias is changed, the potential can vary exclusively inside the ballistic base, and the contacts are excluded from the consideration. ${ }^{10-12}$ The electron gas inside the contacts is assumed to be in thermal equilibrium. However, in contrast to the previous works, ${ }^{10-12}$ the Fermi level in respect to the bottom of the conduction band, denoted here $\varepsilon_{F}$, may take not only negative, but positive values as well, i.e., the injected electrons may be either degenerate or nondegenerate, and follow, in general, the FermiDirac distribution. Assuming the transversal size of the conductor sufficiently thick and high enough electron density, the electrostatic problem is considered in a one-dimensional plane geometry. ${ }^{12}$

\section{DISTRIBUTION FUNCTION AND MEAN CURRENT}

To describe the steady-state transport and low-frequency noise, we use a semiclassical Vlasov system of equations, which consists of the collisionless Boltzmann transport equation for the distribution function and the Poisson equation for the self-consistent electrostatic potential. ${ }^{12,16}$ Due to a stochastic nature of the injection, the distribution function and, consequently, the self-consistent potential both fluctuate in time. The nonuniform distribution of the injected carriers leads to the creation of the potential minimum $\varphi_{m}$ at a position $x=x_{m}$. The potential minimum acts as a barrier for the electrons by reflecting a part of them back to the contact, thereby affecting the transport and noise properties. It is the potential minimum fluctuations that induce the long-range Coulomb interactions and lead to the suppression of the injected current fluctuations. ${ }^{12} \mathrm{We}$ assume that the applied bias $q U>5 k_{B} T$, where $q$ is the electron charge and $T$ is the temperature. From this follows that the current is determined by only one injecting contact (at $x=0$ for definiteness), and the electrons from this contact that are able to pass over the barrier and arrive at the receiving contact at $x=l$ are all absorbed with probability 1 , since the corresponding energy states are empty. All the electrons injected from the receiving contact are reflected back because of the high-bias condition. Their contribution to the current and noise is negligible. Another assumption on the bias is $U_{m} \ll U<U_{c r}$, where $U_{m} \equiv$ $-\varphi_{m}$ is the potential barrier height, and $U_{c r}$ is the bias at which the potential barrier vanishes. ${ }^{12}$ In this limit ("virtualcathode approximation",), only the electrons that are able to pass over the fluctuating barrier contribute to the current and noise. The nonhomogeneous electron density along the ballistic region is determined by ${ }^{16}$

$$
N(x)=\int_{\Phi_{c}}^{\infty} F_{c}(\varepsilon) \frac{d \varepsilon}{2 \sqrt{\varepsilon+\Phi(x)-\Phi_{c}}},
$$

where $\Phi(x)=q \varphi(x)-q \varphi_{m}$ is the mean potential referenced to the minimum, with the value $\Phi_{c} \equiv \Phi(0)$ at the injecting contact. It is clear that in such a definition the contact potential is equal to the potential barrier height, $\Phi_{c}=q U_{m}$. Note that $F_{c}(\varepsilon)$ is the distribution function over the longitudinal kinetic energy $\varepsilon$ at the injecting contact. Since during the ballistic motion only the longitudinal electron momentum may vary, the injection distribution function is averaged over the transversal momentum $\mathbf{k}_{\perp}$ :

$$
F_{c}(\varepsilon)=2 \frac{\sqrt{2 m}}{\hbar} \int \frac{d \mathbf{k}_{\perp}}{(2 \pi)^{d}} f\left(\varepsilon, \mathbf{k}_{\perp}\right),
$$

where $d$ is the dimension of a momentum space, $m$ the electron effective mass, $\hbar$ the Planck constant, $f\left(\varepsilon, \mathbf{k}_{\perp}\right)$ the occupation number of a quantum state, the factor 2 takes into account the spin variable, and the additional factor $\sqrt{2 m} / \hbar$ has been introduced for normalization convenience. Assuming that the number of transversal modes is large, the dimension of a momentum space $d=3$, and we can perform integration over the transversal states. Changing the variable of integration $d \mathbf{k}_{\perp}=\left(2 \pi m / \hbar^{2}\right) d \varepsilon_{\perp}$, where $\varepsilon_{\perp}$ is the transverse electron energy, and taking into account that $f\left(\varepsilon, \varepsilon_{\perp}\right)$ $=f_{F}\left(\varepsilon+\varepsilon_{\perp}\right)$, with $f_{F}(\varepsilon)=\left\{1+\exp \left[\left(\varepsilon-\varepsilon_{F}\right) / k_{B} T\right]\right\}^{-1}$ the Fermi-Dirac distribution, one gets

$$
F_{c}(\varepsilon)=\frac{N_{c}}{\sqrt{\pi k_{B} T}} \ln \left\{1+\exp \left[\left(\varepsilon_{F}-\varepsilon\right) / k_{B} T\right]\right\},
$$

where $N_{c}=2\left(2 \pi m k_{B} T\right)^{3 / 2} /(2 \pi \hbar)^{3}$ is the effective density of states. Integrating the distribution function (3) over the energy, one obtains the electron density injected from the contact,

$$
N_{0}=\int_{0}^{\infty} F_{c}(\varepsilon) \frac{d \varepsilon}{2 \sqrt{\varepsilon}}=\frac{N_{c}}{\sqrt{\pi}} \int_{0}^{\infty} \ln \left(1+e^{\xi-z^{2}}\right) d z
$$

where $\xi \equiv \varepsilon_{F} / k_{B} T$ is the reduced Fermi energy. The injected electron density may also be expressed in a more familiar form

$$
N_{0}=\frac{1}{2} N_{c} \mathcal{F}_{1 / 2}(\xi)
$$

where $\mathcal{F}_{1 / 2}$ is the Fermi-Dirac integral of index $1 / 2$. Since the Fermi-Dirac integrals $\mathcal{F}_{j}$ of different indexes $j$ will be frequently used throughout the paper, their properties are summarized in the Appendix. Note that $N_{0}$ is half of the contact electron density $N_{c} \mathcal{F}_{1 / 2}(\xi)$, since only the electrons with positive momenta are injected into the sample. 


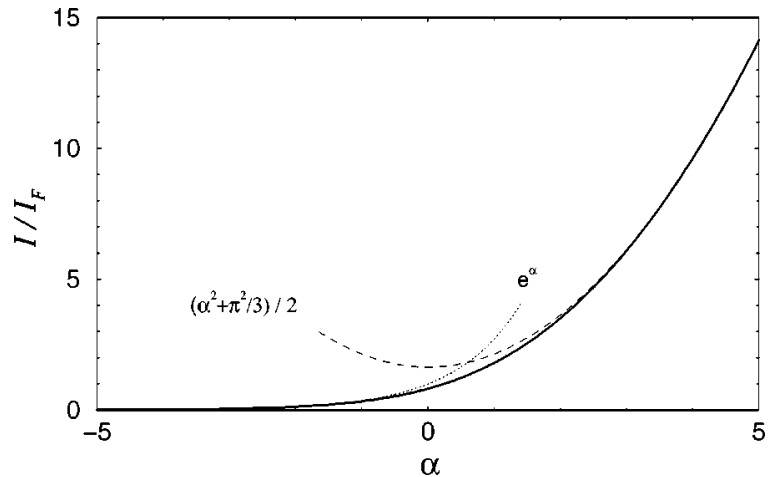

FIG. 1. Current as a function of the position $\alpha$ of the Fermi energy $\varepsilon_{F}$ in respect to the potential barrier $\Phi_{c}, \alpha=\left(\varepsilon_{F}\right.$ $\left.-\Phi_{c}\right) / k_{B} T$. The asymptotic approximations for nondegenerate and degenerate limits are plotted.

Substituting the distribution (3) into Eq. (1) taken at $x$ $=x_{m}$, one obtains the electron density at the potential minimum:

$$
N_{m}=\int_{\Phi_{c}}^{\infty} F_{c}(\varepsilon) \frac{d \varepsilon}{2 \sqrt{\varepsilon-\Phi_{c}}}=\frac{1}{2} N_{c} \mathcal{F}_{1 / 2}(\alpha),
$$

where $\alpha=\left(\varepsilon_{F}-\Phi_{c}\right) / k_{B} T$ is the parameter characterizing the position of the Fermi energy with respect to the potential barrier. The density $N_{m}$ is an important parameter for computing the current noise, as will be seen below.

The steady-state current is obtained by ${ }^{16}$

$$
I=\frac{q A}{\sqrt{2 m}} \int_{\Phi_{c}}^{\infty} F_{c}(\varepsilon) d \varepsilon
$$

where $A$ is the cross-sectional area. Substituting the distribution function (3), one gets

$$
I=I_{F} \int_{0}^{\infty} \ln \left(1+e^{\alpha-y}\right) d y=I_{F} \mathcal{F}_{1}(\alpha),
$$

where $I_{F}=4 \pi q A m\left(k_{B} T\right)^{2} /(2 \pi \hbar)^{3}$ and $\mathcal{F}_{1}$ is the FermiDirac integral (see the Appendix). It is seen that under the ballistic SCL conduction, the current is determined by the relative position of the Fermi energy and the potential barrier through the parameter $\alpha$. This is in contrast to the case of diffusive conductors, in which the current is determined by scattering strength. The parameter $\alpha$ summarizes the dependence of the current on the applied bias and the length of the conductor, since they both affect the potential barrier height, whereas the factor $I_{F}$ is independent of those characteristics.

Figure 1 illustrates the electric current as a function of $\alpha$ given by Eq. (8). When the Fermi energy is sufficiently below the potential barrier, $\alpha<-3$, only the exponential tail of the contact distribution function is injected (nondegenerate injection limit). Under this condition, according to the approximate formulas for the Fermi-Dirac integrals (A5), the current becomes

$$
I \approx I_{F} e^{\alpha} .
$$

When the Fermi energy is above the potential barrier by several $k_{B} T$, it is the degenerate injection limit and, by using Eq. (A6), one gets the approximate formula for the current

$$
I \approx \frac{1}{2} I_{F}\left(\alpha^{2}+\frac{\pi^{2}}{3}\right)
$$

It is seen from Fig. 1, that formula (10) is accurate at $\alpha>2$. Note that the case of nondegenerate injection $\alpha<-3$, may occur when the contact electron density is either nondegenerate or degenerate, depending on the position of the Fermi energy with respect to the conduction band edge characterized by the parameter $\xi$. For $\xi<-3$, the contact electron density is nondegenerate, and this is the case of the MaxwellBoltzmann injection, analyzed in detail in Ref. 12. Let us demonstrate that our formulas are in agreement with that case. Equation (5) gives the injected electron density $N_{0}$ $=\frac{1}{2} N_{c} e^{\xi}$, and the current (9) is expressed as

$$
I=I_{\mathrm{MB}} e^{-U_{m} / k_{B} T},
$$

where $I_{\mathrm{MB}}=I_{F}\left(2 N_{0} / N_{c}\right)=q A N_{0} \sqrt{2 k_{B} T / \pi m}$ is the emission current for the Maxwell-Boltzmann distribution [compare with Eq. (46) of Ref. 12]. For $\xi>-3$ and $\alpha<-3$, the injected electrons that pass over the barrier are nondegenerate, but the contact electrons are degenerate; hence the approximate formula (11) for the current is no longer valid, and one has to use a more general relation, Eq. (9).

It should be also noted that in the general case of a FermiDirac injection, the contact emission current is $I_{0}=I_{F} \mathcal{F}_{1}(\xi)$. This is the maximum (saturation) current that is achieved when the applied bias $U \geqslant U_{c r}$, the barrier vanishes $\left(\Phi_{c}\right.$ $=0, \alpha=\xi)$, and the conduction is no longer space-charge limited. The current in units of its saturation value is simply

$$
\frac{I}{I_{0}}=\frac{\mathcal{F}_{1}(\alpha)}{\mathcal{F}_{1}(\xi)} .
$$

It was shown in a previous paper ${ }^{16}$ that the asymptotic behavior of the current in SCL ballistic conductors obeys the Child law in the leading-order terms independently of the injection distribution:

$$
I_{\text {Child }}=\frac{4}{9} \kappa A \sqrt{\frac{2 q}{m}} \frac{U^{3 / 2}}{l^{2}},
$$

where $\kappa$ is the dielectric permittivity, and $l$ is the length of the ballistic conductor. However, this formula is only accurate at very high biases, in the range where the SCL conduction is difficult to maintain. This is a consequence of a rough approximation, in which the velocity spread of electrons at the potential minimum is neglected. To obtain a satisfactory good approximation at lower biases, it is necessary to keep the next-order terms that are specific with respect to the injection distribution. The general formula for an arbitrary injection function has been recently derived: ${ }^{16}$ 


$$
I=I_{\text {Child }}\left(1+\frac{3}{\sqrt{q U}} \frac{\int_{0}^{\infty} F_{c}\left(\epsilon+\Phi_{c}\right) \epsilon^{1 / 2} d \epsilon}{\int_{0}^{\infty} F_{c}\left(\epsilon+\Phi_{c}\right) d \epsilon}\right) .
$$

In our case of the injection distribution function (3), one finds the following expression:

$$
I=I_{\text {Child }}\left(1+\frac{3 \sqrt{\pi}}{2} \sqrt{\frac{k_{B} T}{q U}} \frac{\mathcal{F}_{3 / 2}(\alpha)}{\mathcal{F}_{1}(\alpha)}\right) .
$$

In the nondegenerate limit, $\alpha<-3$, one obtains $\left(\mathcal{F}_{3 / 2} / \mathcal{F}_{1}\right)$ $\rightarrow 1$, and Eq. (15) leads to the Langmuir formula for the Maxwell-Boltzmann injection. ${ }^{12}$ In the opposite limit of high degeneracy, $\alpha \gg 1$, one gets

$$
I \approx I_{\text {Child }}\left(1+\frac{8}{5} \sqrt{\frac{\varepsilon_{F}-q U_{m}}{q U}}\right),
$$

which can be used to estimate the current for Fermi ballistic conductors beyond the Child approximation. Here, we remark that in the degenerate limit and at $\varepsilon_{F} \gg q U_{m}$, the current (16) is independent of temperature $T$ in both terms. For an arbitrary degree of degeneracy, the general expression (15) can be used.

\section{CURRENT NOISE}

To calculate the current noise, one has to define the partial injection current $I_{c}(\varepsilon)$ at the contact and its fluctuation properties. From Eq. (8) it follows that

$$
I_{c}(\varepsilon)=\frac{I_{F}}{k_{B} T} \ln \left[1+e^{\left(\varepsilon_{F}-\varepsilon\right) / k_{B} T}\right],
$$

which corresponds to the current carried by electrons with injection (longitudinal) energies between $\varepsilon$ and $\varepsilon+d \varepsilon$, giving after the integration the total emission current $I_{0}$ $=\int_{0}^{\infty} I_{c}(\varepsilon) d \varepsilon$.

The correlation function for the fluctuations of the partial injection currents may be written generally as

$$
\left\langle\delta I_{c}(\varepsilon) \delta I_{c}\left(\varepsilon^{\prime}\right)\right\rangle=K(\varepsilon)(\Delta f) \delta\left(\varepsilon-\varepsilon^{\prime}\right),
$$

where $\Delta f$ is the frequency bandwidth (we assume the lowfrequency limit). For the particular case of Fermi 3D injection, the function $K(\varepsilon)$ is determined by ${ }^{17,18}$

$$
K(\varepsilon)=2 q \frac{2 q A}{\hbar} \int \frac{d \mathbf{k}_{\perp}}{(2 \pi)^{d}} f\left(\varepsilon, \mathbf{k}_{\perp}\right)\left[1-f\left(\varepsilon, \mathbf{k}_{\perp}\right)\right] .
$$

The integration over the transversal states may be performed explicitly by taking into account that $f_{F}\left(1-f_{F}\right)=$ $-k_{B} T\left(\partial f_{F} / \partial \varepsilon\right) \quad$ and $\int_{0}^{\infty} d \varepsilon_{\perp} f_{F}\left(\varepsilon+\varepsilon_{\perp}\right)\left[1-f_{F}\left(\varepsilon+\varepsilon_{\perp}\right)\right]$ $=k_{B} T f_{F}(\varepsilon)$. This gives a simple expression:

$$
K(\varepsilon)=\frac{2 q I_{F}}{k_{B} T} f_{F}(\varepsilon) .
$$

We remind the reader that $\varepsilon$ is the longitudinal energy component. The Fermi factor $1-f_{F}$ has disappeared after the

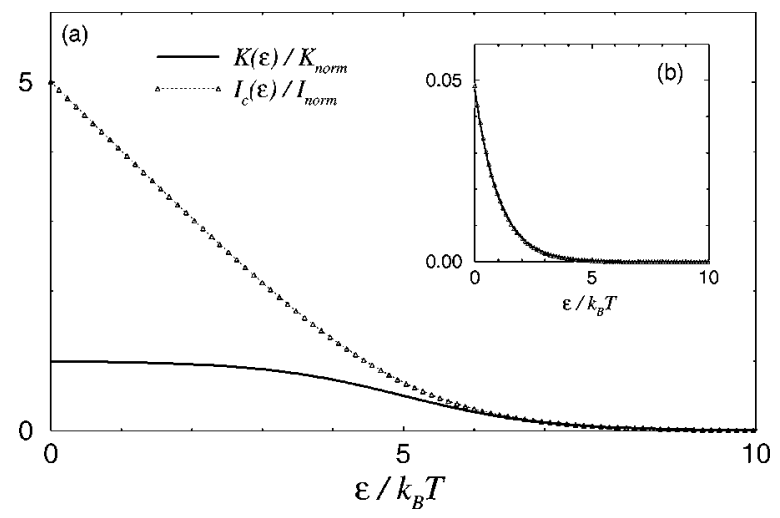

FIG. 2. Shot-noise power per unit energy $K(\varepsilon)$ and partial current $I_{c}(\varepsilon)$ for injected electrons for two cases: (a) degenerate, $\xi=5$; (b) nondegenerate, $\xi=-3$. Here, $K_{\text {norm }}=2 q I_{F} / k_{B} T, \quad I_{\text {norm }}$ $=I_{F} / k_{B} T$.

integration, ${ }^{19}$ but the Fermi noise-suppression effect is present in Eq. (20). Indeed, in the degenerate limit $\varepsilon_{F}$ $\gg k_{B} T$, the partial current (17) is a linear function of energy, $I_{c}(\varepsilon) \sim\left(\varepsilon_{F}-\varepsilon\right)$, at $\varepsilon \rightarrow 0$ [see Fig. 2(a)]. This occurs because of the increase of the number of transversal states as the longitudinal energy $\varepsilon$ decreases. Despite the increasing of the number of states, the shot-noise power per unit energy represented by the function (20) is constant at $\varepsilon \ll \varepsilon_{F}$ [Fig. 2 (a) $]$. As a result, $K(\varepsilon) / 2 q I_{c}(\varepsilon) \approx 1 / \xi \ll 1$ at $\varepsilon \rightarrow 0$, indicating the noise suppression effect. In contrast, for nondegenerate case, both functions $\propto \exp \left[\left(\varepsilon_{F}-\varepsilon\right) / k_{B} T\right]$ and $K(\varepsilon) / 2 q I_{c}(\varepsilon)$ $\approx 1$ [Fig. 2(b)], which leads to the Poisson noise. Additionally, we note that, since $I_{F} \sim T^{2}$, the injection noise vanishes, $K(\varepsilon) \rightarrow 0$, in the limit $T \rightarrow 0$.

The current-noise spectral density for the electron flow, when Coulomb correlations are disregarded, is given by ${ }^{16}$

$$
S_{I}^{\text {uncor }}=\int_{\Phi_{c}}^{\infty} K(\varepsilon) d \varepsilon
$$

Here, the integration is performed over the energies above the barrier height $\Phi_{c}$, since only the electrons transmitted over the barrier contribute to the current noise at high biases. Substitution of expression (20) yields

$$
S_{I}^{\text {uncor }}=2 q I_{F} \ln \left(1+e^{\alpha}\right)=2 q I_{F} \mathcal{F}_{0}(\alpha) .
$$

From this result we find the shot-noise-suppression factor caused by Fermi correlations

$$
\Gamma_{F}=\frac{S_{I}^{\text {uncor }}}{2 q I}=\frac{\mathcal{F}_{0}(\alpha)}{\mathcal{F}_{1}(\alpha)} .
$$

This function is plotted in Fig. 3. It is clear that in the nondegenerate limit, one gets $\left(\mathcal{F}_{0} / \mathcal{F}_{1}\right) \rightarrow 1$, and obviously $\Gamma_{F}$ $\rightarrow 1$. An important feature is that $\Gamma_{F}$ is a decreasing function of $\alpha$. In the degenerate limit, $\alpha \gg 1$, it approaches the asymptotic behavior:

$$
\Gamma_{F} \approx \frac{2}{\alpha+\left(\pi^{2} / 3 \alpha\right)} .
$$




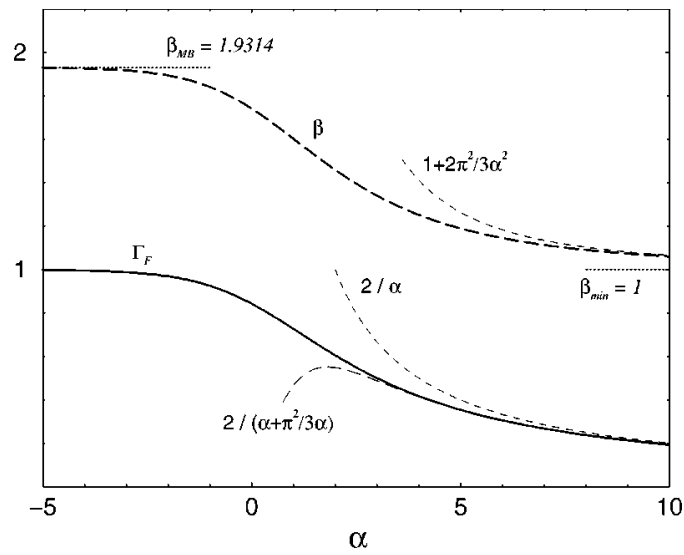

FIG. 3. Fermi shot-noise-suppression factor $\Gamma_{F}$ and shot-noise parameter $\beta$ as functions of the position of the Fermi energy $\alpha$. The asymptotic approximations for nondegenerate and degenerate limits are shown.

It is seen from Fig. 3 that formula (24) is accurate at $\alpha>3$. The limiting minimal value for $\Gamma_{F}$ occurs when the barrier vanishes $(\alpha=\xi)$,

$$
\Gamma_{F}^{\min }=\frac{2}{\xi}=\frac{2 k_{B} T}{\varepsilon_{F}} .
$$

The numerical factor in Eq. (25) depends on the dimensionality of a momentum space. By taking different values of $d$ in Eqs. (2) and (19), one can get $\Gamma_{F}^{\mathrm{min}}=c k_{B} T / \varepsilon_{F}$ with $c=2$ $(d=3), 3 / 2(d=2)$, and $1(d=1)$. In all the cases, the shotnoise Fermi suppression is determined by the ratio between the temperature of the injected electrons $T$ and their Fermi energy $\varepsilon_{F}$. For a fixed $\varepsilon_{F}$, the suppression may be enhanced by decreasing the temperature $T \rightarrow 0$, but it is independent of the bias, the ballistic length and the other parameters of the conductor.

The current noise, which takes into account both Fermi and Coulomb correlations, is determined by ${ }^{16}$

$$
S_{I}=\int_{\Phi_{c}}^{\infty} \gamma^{2}(\varepsilon) K(\varepsilon) d \varepsilon,
$$

where the energy-resolved shot-noise-suppression factor

$$
\gamma(\varepsilon)=\frac{3}{\sqrt{q U}}\left[\sqrt{\varepsilon-\Phi_{c}}-v\right],
$$

and the constant $v$ for an arbitrary injection distribution is given by ${ }^{16}$

$$
v=\frac{N_{m}}{F_{c}\left(\Phi_{c}\right)} .
$$

By using Eqs. (3) and (6), we find for the Fermi 3D injection

$$
v=\frac{\sqrt{\pi k_{B} T}}{2} \frac{\mathcal{F}_{1 / 2}(\alpha)}{\mathcal{F}_{0}(\alpha)} .
$$

Thus, for the current-noise power (26), after using Eqs. (20), (27), and (29), we find

$$
S_{I}=\beta 2 q I \frac{k_{B} T}{q U} .
$$

In this formula, the constant $\beta$ is determined only by $\alpha$ :

$$
\beta(\alpha)=9\left(1-\frac{\pi}{4} \frac{\left[\mathcal{F}_{1 / 2}(\alpha)\right]^{2}}{\mathcal{F}_{0}(\alpha) \mathcal{F}_{1}(\alpha)}\right) .
$$

To distinguish the noise suppression caused by different mechanisms, one can define the shot-noise-suppression factor due to a pure Coulomb suppression

$$
\Gamma_{C}=\frac{S_{I}}{S_{I}^{\text {uncor }}}=\beta \frac{k_{B} T}{q U} \frac{\mathcal{F}_{1}(\alpha)}{\mathcal{F}_{0}(\alpha)},
$$

whereas the overall shot-noise-suppression factor becomes

$$
\Gamma=\Gamma_{C} \Gamma_{F}=\frac{S_{I}}{2 q I}=\beta \frac{k_{B} T}{q U} .
$$

It is seen that the current noise may be suppressed by both the temperature $T$ and the bias $U$. This is in contrast to the pure Fermi suppression (25), which is sensitive to $T$, but independent of the bias. The dependence on $U$ comes from the Coulomb correlations and originates from the function $\gamma(\varepsilon)$. The coefficient $\beta$ is a parameter that depends on the degree of degeneracy, as follows from Eq. (31). For the Fermi 3D injection, $\beta$ is a decreasing function of $\alpha$ ranging between two limiting values (see Fig. 3): $\beta_{\min }<\beta<\beta_{\mathrm{MB}}$, where $\beta_{\mathrm{MB}}=9(1-\pi / 4) \approx 1.9314$ is a limiting value in the nondegenerate limit (Maxwell-Boltzmann injection), and $\beta_{\min }=1$ is a limiting value in the degenerate limit. For high degeneracy, the approximate formula for $\beta$ may be obtained by using the expansions for the Fermi-Dirac integrals (A6), one gets

$$
\beta \approx 1+\frac{2}{3} \frac{\pi^{2}}{\alpha^{2}}, \quad \alpha \gg 1 .
$$

Figure 3 demonstrates the validity of such an approximation. The asymptotic behavior of the Coulomb suppression factor $\Gamma_{C}$ in a high degenerate limit is obtained as

$$
\Gamma_{C} \approx \frac{1}{2} \frac{\varepsilon_{F}-\Phi_{c}}{q U}, \quad \varepsilon_{F}-\Phi_{c} \gg k_{B} T,
$$

which takes the minimal value at $U=U_{c r}$ :

$$
\Gamma_{C}^{\min } \approx \frac{\varepsilon_{F}}{2 q U_{c r}} .
$$

It should be emphasized that the difference between the two noise-suppression mechanisms is fundamental: While $\Gamma_{F}$ cannot be decreased further by varying the parameters of the conductor, since its minimal value is fixed by the contact properties [by the parameter $\xi$ as follows from Eq. (25)]. In contrast, the factor $\Gamma_{C}$ may be decreased by increasing the ballistic length $l$ of the conductor, since for longer conductors the critical bias $U_{c r}$ under which the barrier disappears is higher, and $\Gamma_{C}$ may drop deeper. As a consequence, $\Gamma$ may also attain much lower values. It is important to highlight, 


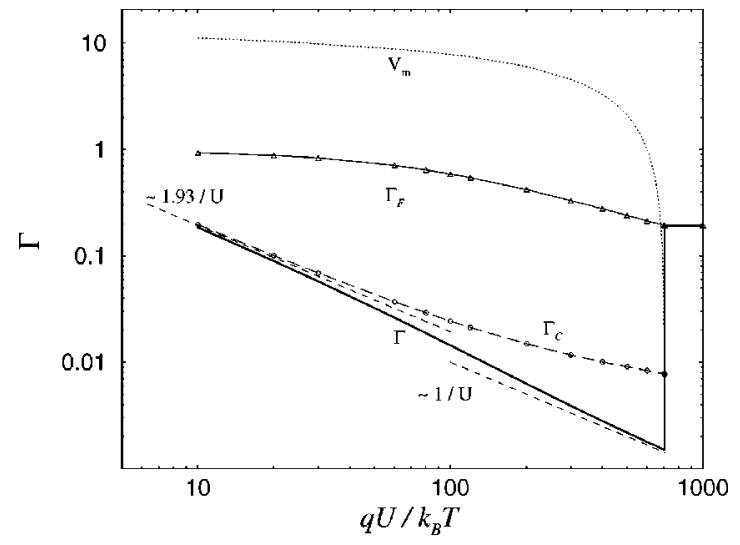

FIG. 4. Shot-noise-suppression factors: $\Gamma_{F}$ (Fermi), $\Gamma_{C}$ (Coulomb), and $\Gamma=\Gamma_{F} \Gamma_{C}$ (total), and potential barrier height $V_{m}$ as functions of applied bias $U$. The asymptotic limiting lines $\beta_{M B}\left(k_{B} T / q U\right)$ and $k_{B} T / q U$ for $\Gamma$ are shown by dashes.

that in both nondegenerate and degenerate limits, the total shot-noise suppression factor $\propto k_{B} T$, and can therefore be reduced by decreasing the temperature.

\section{EXAMPLE}

To illustrate the results, consider the GaAs $n-i-n$ ballistic diode of length $l=0.5 \mu \mathrm{m}$ at $T=4 \mathrm{~K}$. For this temperature and $m=0.067 m_{0}$, the effective density of states is $N_{c}$ $\approx 6.7 \times 10^{14} \mathrm{~cm}^{-3}$. Assuming the contact doping 1.6 $\times 10^{16} \mathrm{~cm}^{-3}$, the reduced Fermi energy $\xi \approx 10$, and the contact electrons are degenerate. For this set of parameters, the Debye screening length associated with the contact degenerate electron density is approximately $L_{D}$ $=\sqrt{\kappa k_{B} T /\left[q^{2} N_{c} \mathcal{F}_{-1 / 2}(\xi)\right]} \approx 14 \mathrm{~nm}$. Since $L_{D} \ll l$, the spacecharge effects and, therefore, the Coulomb shot-noise suppression are important in a wide range of biases.

Let us introduce the normalized biases: $V=q U / k_{B} T$ and $V_{m}=q U_{m} / k_{B} T$. The calculation of the steady-state potential profile for different biases $V$ shows that the potential barrier varies from $V_{m} \approx 11.2$ at $V=10$ to $V_{m}=0$ at $V=V_{c r} \approx 705$ ( $U_{c r} \approx 243 \mathrm{mV}$ ) (see Fig. 4). In this range, the chargelimited conduction is controlled by the barrier height $V_{m}$, and by increasing the bias, one can observe the crossover from nondegenerate $\left(\alpha=\xi-V_{m}<-1\right)$ to degenerate (2 $<\alpha<10$ ) injection. This crossover is illustrated in Fig. 4, where the shot-noise suppression factors $\Gamma, \Gamma_{F}$, and $\Gamma_{C}$ are plotted as functions of bias. Indeed, the Fermi suppression factor $\Gamma_{F}$ varies from 1 at low biases to $2 / \xi \approx 0.2$ at high biases, in agreement with formulas (23)-(25). Moreover, the factor $\Gamma$ lies between two asymptotic lines: $\beta_{\mathrm{MB}}\left(k_{B} T / q U\right)$ at low biases (nondegenerate limit) and $k_{B} T / q U$ at high biases (degenerate limit), in agreement with Eq. (33) and the variation of $\beta$ in Fig. 3. The Coulomb correlation factor $\Gamma_{C}$ decreases with bias up to the lowest value $\approx 0.0078$ at $U$ $=U_{c r}$. After that value it increases sharply to 1 due to the disappearance of the potential barrier. The sharp increase of $\Gamma_{C}$ at $U=U_{c r}$ is discontinuous in this asymptotic theory, which neglects the high-order terms in the expansions. The exact calculations ${ }^{20}$ give a smoother behavior. Note that both mechanisms essentially suppress shot noise at large $U$, but $\Gamma_{C}$ is always much lower than $\Gamma_{F}$ under SCL conditions.

\section{SUMMARY}

In conclusion, we have derived the analytical formulas that describe the mean current and the shot-noise power in degenerate space-charge-limited ballistic conductors. In the framework of a semiclassical Vlasov system of equations, which takes into account the fluctuations of the potential profile self-consistently, we have obtained a deep shot-noise suppression of more than two orders of magnitude caused by two independent mechanisms: Fermi and Coulomb correlations. The derived formulas clearly distinguish the shot-noise suppression factors caused separately by Fermi correlations (23), Coulomb correlations (32), and by the joint action of both [Eq. (33)].

We show that the Fermi shot-noise-suppression factor is limited below by the ratio between the temperature and Fermi energy of the contact electrons. The Coulomb noisesuppression factor, however, may attain much lower values $\varepsilon_{F} / 2 q U$, because of its dependence on the applied bias $U$ $\gg k_{B} T / q$. The asymptotic behavior of the overall shot-noisesuppression factor in a high degenerate limit was found to be $k_{B} T / q U$, independently of the material parameters. Finally, for the degenerate Fermi-Dirac injection, the asymptotic formula for the mean current beyond the Child approximation is proposed. $^{21}$

\section{ACKNOWLEDGMENTS}

We are grateful to V. A. Kochelap for valuable discussions. This work has been partially supported by the Generalitat de Catalunya, Spain, and the NATO linkage Grant No. HTECH.LG 974610.

\section{APPENDIX: FERMI-DIRAC FUNCTIONS AND THEIR APPROXIMATIONS}

The Fermi-Dirac functions are encountered, whenever one wants to describe the electronic transport in degenerate semiconductor or metallic systems, and they are defined $a^{22}$

$$
\mathcal{F}_{j}(\alpha)=\frac{1}{\Gamma(j+1)} \int_{0}^{\infty} \frac{y^{j} d y}{1+e^{y-\alpha}},
$$

where $\Gamma(j)$ is the gamma function of the index $j$. For the expressions of this paper, the $\Gamma$ functions take the values $\Gamma\left(\frac{3}{2}\right)=\sqrt{\pi} / 2, \Gamma\left(\frac{5}{2}\right)=3 \sqrt{\pi} / 4, \Gamma(1)=\Gamma(2)=1$.

For positive indexes $j$, the Fermi-Dirac integrals can also be rewritten [obtained by integrating by parts (A1)]:

$$
\mathcal{F}_{j}(\alpha)=\frac{j}{\Gamma(j+1)} \int_{0}^{\infty} y^{j-1} \ln \left(1+e^{\alpha-y}\right) d y .
$$

A simple relation between the integrals of different orders is

$$
\mathcal{F}_{j}(\alpha)=d \mathcal{F}_{j+1} / d \alpha
$$


Unfortunately, these integrals cannot be resolved analytically except for the trivial cases:

$$
\begin{gathered}
\mathcal{F}_{0}(\alpha)=\ln \left(1+e^{\alpha}\right), \quad j=0 \\
\mathcal{F}_{-1}(\alpha)=\left(1+e^{-\alpha}\right)^{-1}, \quad j=-1 .
\end{gathered}
$$

However, for small and large $\alpha$, one may use the approximate formulas ${ }^{22}$ for the nondegenerate limit, $\alpha<-2$,

$$
\mathcal{F}_{j}(\alpha)=e^{\alpha} \quad \forall j,
$$

and for the degenerate limit, $\alpha \gg 1$,

$$
\mathcal{F}_{j}(\alpha)=\frac{\alpha^{j+1}}{(j+1) \Gamma(j+1)}\left[1+\frac{\pi^{2}}{6} \frac{j(j+1)}{\alpha^{2}}+O\left(\frac{1}{\alpha^{4}}\right)\right]
$$

${ }^{1}$ M.J.M. de Jong and C.W.J. Beenakker, in Mesoscopic Electron Transport, edited by L.P. Kowenhoven, G. Schön, and L.L. Sohn (Kluwer, Dordrecht, 1997), p. 225.

${ }^{2}$ Ya.M. Blanter and M. Büttiker, Phys. Rep. 336, 1 (2000).

${ }^{3}$ G.B. Lesovik, Pis'ma Zh. Éksp. Teor. Fiz. 49, 513 (1989) [JETP Lett. 49, 592 (1989)].

${ }^{4}$ M. Büttiker, Phys. Rev. Lett. 65, 2901 (1990); Phys. Rev. B 46, 12485 (1992).

${ }^{5}$ R. Landauer and Th. Martin, Physica B 175, 167 (1991); Th. Martin and R. Landauer, Phys. Rev. B 45, 1742 (1992).

${ }^{6}$ Y.P. Li, D.C. Tsui, J.J. Heremans, J.A. Simmons, and G.W. Weimann, Appl. Phys. Lett. 57, 774 (1990).

${ }^{7}$ M. Reznikov, M. Heiblum, H. Shtrikman, and D. Mahalu, Phys. Rev. Lett. 75, 3340 (1995).

${ }^{8}$ A. Kumar, L. Saminadayar, D.C. Glattli, Y. Jin, and B. Etienne, Phys. Rev. Lett. 76, 2778 (1996).

${ }^{9}$ H.E. van den Brom and J.M. van Ruitenbeek, Phys. Rev. Lett. 82, 1526 (1999).

${ }^{10}$ T. González, O.M. Bulashenko, J. Mateos, D. Pardo, and L. Reggiani, Phys. Rev. B 56, 6424 (1997).

${ }^{11}$ O.M. Bulashenko, J. Mateos, D. Pardo, T. González, L. Reggiani, and J.M. Rubí, Phys. Rev. B 57, 1366 (1998).

${ }^{12}$ O.M. Bulashenko, J.M. Rubí, and V.A. Kochelap, Phys. Rev. B 61, 5511 (2000).

${ }^{13}$ K.K. Likharev, Proc. IEEE 87, 606 (1999).

${ }^{14}$ Y. Naveh, A.N. Korotkov, and K.K. Likharev, Phys. Rev. B 60, R2169 (1999).
${ }^{15}$ T. González, J. Mateos, D. Pardo, and L. Reggiani, Physica B 272, 285 (1999).

${ }^{16}$ O.M. Bulashenko, J.M. Rubí, and V.A. Kochelap, Phys. Rev. B 62, 8184 (2000).

${ }^{17}$ Sh. Kogan, Electronic Noise and Fluctuations in Solids (Cambridge University Press, Cambridge, 1996), Chap. 5.

${ }^{18}$ Expression (19), when rewritten as $K(\varepsilon)=2 q I_{c}(\varepsilon)[1-R(\varepsilon)]$, with $I_{c}(\varepsilon)=2 q A /\left[(2 \pi)^{d} \hbar\right] \int f\left(\varepsilon, \mathbf{k}_{\perp}\right) d \mathbf{k}_{\perp} \quad$ and $\quad R(\varepsilon)$ $=\left[\int f^{2}\left(\varepsilon, \mathbf{k}_{\perp}\right) d \mathbf{k}_{\perp}\right] /\left[\int f\left(\varepsilon, \mathbf{k}_{\perp}\right)\right] d \mathbf{k}_{\perp}$, coincides with Eq. (2) given by Naveh et al. (Ref. 14).

${ }^{19}$ The disappearance of the factor $1-f_{F}$ in Eq. (20) leads to a remarkable difference between the multichannel $3 \mathrm{D}$ case and a one-channel case studied earlier (Ref. 5). In a 1D conductor the noise comes from a narrow energy range of order $k_{B} T$ at the Fermi level, while in a 3D conductor the main contribution to the noise comes from the lowest longitudinal energies far below the Fermi energy, where more transversal states within the range $k_{B} T$ near the Fermi sphere are available [see also discussion of Fig. 2(a)].

${ }^{20}$ O.M. Bulashenko and J. M. Rubí (unpublished).

${ }^{21}$ Note that in all the asymptotic formulas, for both the current and noise, the bias $U$ may be replaced by the more exact $U+U_{m}$. Because of our assumption $U_{m} \ll U$, we neglect $U_{m}$. This correction may improve, however, the results at intermediate biases when the barrier is not sufficiently small.

${ }^{22}$ J.S. Blakemore, Semiconductor Statistics (Dover, New York, 1982). 